\title{
BNDNN: Batch Normalization Based Deep Neural Network for Predicting Flood in Urban Areas
}

\section{Vinay Dubey}

Delhi Technological University

Rahul Katarya ( $\nabla$ rahuldtu@gmail.com )

Delhi Technological University https://orcid.org/0000-0001-7763-291X

\section{Research Article}

Keywords: Artificial Neural Network, Batch Normalization, Decision Tree, Deep Neural Network, Flood prediction, Support Vector Machine

Posted Date: November 1st, 2021

DOI: https://doi.org/10.21203/rs.3.rs-1027530/v1

License: (c) (1) This work is licensed under a Creative Commons Attribution 4.0 International License. Read Full License 


\section{BNDNN: Batch Normalization based Deep Neural 2 Network for predicting flood in urban areas}

\section{Abstract}

Vinay Dubey, Rahul Katarya

Big Data Analytics and Web Intelligence Laboratory, Delhi technological

University, New Delhi, India

vinaydubeyamit@gmail.com, rahuldtu@gmail.com

Disaster is a very serious dissipation that arises for a short time period, but the impact of that disaster on human society is very dangerous and very long-lasting. Disasters are categorized into two types like natural disasters and manmade disasters. Among all disasters, of all the natural disasters, flood is the commonplace natural disaster. Flood disaster that causes huge loss of human life, diversity as well as economic loss, which is very dangerous for the developing countries and developed countries also. Nowadays during the monsoon season flood is dangerous for all the geographical areas located nearby water bodies. Much research has been done for flood detection. Machine Learning and many other recent technologies are playing a vital role in predicting the occurrence of floods. For prediction purposes, a huge amount of data is requiring collected from sensors deployed in various locations. In this paper, we used the Batch normalization with Deep Neural Network (BNDNN) technique for the classification of data in three classes as Low, Moderate, and High. The result obtained from our proposed model is compared with some other models like Decision Tree (DT), Support Vector Machine (SVM), Artificial Neural Network (ANN), and Deep Neural Network (DNN). In this our proposed BNDNN provides $89 \%$ accuracy which is higher among all existing models. Models are compared based on some parameters like Accuracy, Precision, Recall, F Score. The compression among all the models used in this paper shows that our proposed model provides better results.

Key Words: Artificial Neural Network; Batch Normalization; Decision Tree; Deep Neural Network; Flood prediction; Support Vector Machine

\section{Introduction}

Disaster is a very serious outbreak that is of very short duration but has a huge impact on human life and nature. According to the united nation, a disaster is

A disaster can be defined as a "serious severance of a biodiversity or society's working due to widespread human or environmental damage". 
Nowadays, technologies are sufficient to predict the severity of the disaster such as flood and also detect disaster in inaccessible areas. Flood risk residential life and property, altering the natural environment, profound impact on biodiversity, polluting water bodies and ecosystem [Hirabayashi et al., 2013, Dottori et al., 2016] Nevertheless, a huge number of studies and explorations are done on sensor technology and artificial intelligence. The combination of these vital areas provides immense business value and a huge number of conveniences for burgeoning artificial intelligence technology and big data analytics. Monitoring discrete events is important, but our main goal is to process data collected from deployed sensory sources. Change in the climate of the earth is contributing to increasing weather-related events. That encounters the increment in natural disasters like floods, earthquakes, and tsunami, etc. [Mosavi et al., 2018]. Out of all the disasters, flood is the most frequent one. The reason behind floods is sometimes natural such as excessive rainfall or cloudburst or sometimes man-made such as encroachment of water bodies in the catchment areas.

Flesh flood disaster is referring to loss that is influenced by the flood in the form of causalities, infrastructure loss, and diversity loss as well as economical loss. For developing countries, any natural disaster is very destructive [Li et al., 2019]. Flood disaster very commonly occurs in natural disasters among all-natural disasters such as earthquakes, volcanoes, tsunami, and landslides. The impact of these disasters on human life is very extensive [Liu et al. 2019]. According to a survey, statistical economic loss due to the flood annually around the world for 50 million dollars approximately. The people concerned by the flood are approximate 100 million [Ahmadisharaf et al. 2015]. However, detection and prediction of the flooded area are required time and it is a very complex task due to the dynamically change in the climate stage. So thus nowadays mostly data-oriented flood prediction models are developed and concern in very uncomplicated presumptions [Aazam et al. 2014]. The unceasing improvement of Machine Learning approaches over the past decades determined their appropriateness for flood detection with the plausible rate of outperforms conventional approaches [Saeed et al. 2018, Ranit and Durge et al. 2019]. We have used DNN to classify our input data collected from different sensors. We add batch normalization techniques between layers to improve the classification accuracy of our DNN model. The accuracy of our model is better than the accuracy previously used for flood prediction.

In the current section of the paper, we provide the basic introduction of the flood disaster as well as some statistical data regarding the flood. These sections also highlight the basic information of the model which we use in our paper. Paper flow is in section 2, we provide a literature survey. In this section, we discuss the previously used model and algorithm used in flood prediction. In section 3, We have discussed some of the standard algorithms and models used earlier in flood prediction. In section 4, we discuss our proposed model for flood prediction. In section4, we provide information regarding the 
data set we used in our paper as well as compare our model with some other models. In section 5, we provide the conclusion of the paper as well we the future scope.

Our major contribution in this paper:

- In this paper, we introduce the new approach, where the batch normalization approach combines with DNN for meat flood event prediction.

- The accuracy of our model is also compared to some existing standard models and algorithms and our proposed model provides better prediction accuracy.

- In this paper, we consider datasets with different environmental parameters, such as cloud cover, precipitation, average. temp, min. temp, max. temp. along with this parameter year, the month is also included.

\section{Related work}

For reducing the risk of possible damage caused by the disaster government all over the world focus on developing the Early Warning System (EWS). The author develops an ML method for monitoring flood areas. The AI-based system is designed for detecting abnormal behavior of dike. The Bishop model was used to calculate the Dike behavior and used as a target for the neural network [Pyayt et al., 2011]. Prediction of drainage of water is crucial for flood control during heavy rainfall. The proposed work focuses on the predictive discharge BPNN model. Data is collected from Govindpur basins on the Brahmani River on daily basis. Models have been Trained and tested to predict model performance over different architectures [Ghose et al. 2018]. The author uses the Hadoop file system to maintain a huge amount of data collected from sensors. a Convolution DNN for the analysis of data. Model is tested on data collected from IoT-based devices. Results were compared with the existing ANN and DNN models. [Anbarasan et al. 2020]. IoT is an area of applied electronics that is deals with collecting data in a real-time environment and transfer via a wireless sensor network. Data collected from the various sensors is analyzed through an ANN. In this proposed model standard three-layer architecture is used with one hidden layer [Mitra et al. 2016]

The study area in this paper is disaster risk analysis in the Philippines due to cyclones. The researcher in this study is the focus to improve the output of the hybrid model. This model uses ARIMA and ANN with DWT for disaster risk prediction in the Philippines provinces in terms of casualties [Alquisola et al. 2018]. The existing tool for data classification is mostly used manual data input and prepared data. The author proposed a unique approach for flood forecasting by integrating geospatial, Hydrological, Metrological, and crowdsources that big data in an appropriate Machine Learning framework. Results suggest that MLP ANNs provide better accuracy among all ML techniques [Puttinaovarat et al. 2020]. Flood occurrence is depending on various 
metrological and hydrological factors. An ANN-based model aims to intensify the accuracy of the flood detection system. Data from various sensors fed to the ANN for analysis. Experimental results show that the NARX network with Levenberg- Marquardt training algorithm provides better results and real-time prediction with alert [Bande et al. 2017].

In many cases, ANN is providing better prediction accuracy. Japan is the very venerable geographical area for floods, which facing an increase in water level after Typhoon. In this paper, the author used various datasets. This work aims to select the most relevant dataset for the ANN-based water flow forecast model [Kim et al. 2016]. The study area in this paper is the Pampanga river basin. The hardware setup contains a microcontroller, solar penal, ultrasonic sensor, and GSM module. A Feedforward network with backpropagation is used and for optimizing the network Levenberg- Marquardt training algorithm is used [Sahagun et al. 2017]. In some cases, a tree-based ML model is also used to predict the sensitivity of flooded areas based on the spatial parameters [Lee et al. 2017]. A novel framework is design by the author to detect the flooded area and depth of flood by using ML measures and a hybrid of hydraulic models. To achieve this a twodimensional hydraulic model (iRIC), calibrated by continuous water level data, is used to estimate river depth by applying a two ML model to the domain for an arbitrary discharge. is done to train. [Hosseiny et al. 2020]. Nowadays IoT technology is very commonly used for real-time data. The author proposed IoT based approach, to cover wide-area and reliability LoRaWAN method is used. For forecasting the occurrence of flood GRU neural network, LASTM and ANN model is used [Mousavi et al. 2021].

For reducing the flood damage, An ANN is used to predict the flood in Sungai, Malaysia. Three different optimization algorithms named LM, back-propagation, GD, and BR with back-propagation for optimizing the ANN result. The result of the prediction of BR is satisfactory [Keong et al. 2016]. Eight different ML models are implemented and their results were compared. 201 flood events are included in this dataset and 10,000 were randomly selected in the Haraz watershed (Iran) Non-event number. Among all models, The proposed EMmedian provides a higher accuracy [Shafizadeh-Moghadam et al. 2018].

Neural network training is a very tedious task as well as computationally expensive. To reduce the complexity of training time normalizes the activation of neurons. In the latest research Batch normalization technique was used when training our network. The efficiency of batch normalization is depending on the mini-batch size. In this paper, the author uses the layer normalization concept to speed up NN training [Al Nuaimi et al. 2015]. In a neural network, the input of each layer depends on the output of the preceding layer, a small change can lead to a big difference. The batch normalization technique is used to solve this by normalizing for mini-batch. This paper is focused on BN for physical buckling. The author design the $\mathrm{CNN}$ model with or without batch 
normalization and tested that modal on the MNIST dataset [Chen et al. 2017]. In this paper author efficiently us the concert of batch normalization for improving the speed of DNN. To calculate the effectiveness of the method results are validate on CPU, GPU, and Raspberry Pi. An experiment was done by using the Caffe framework by combining the batch normalization and previous liner layer [Duan et al. 2018].

Recurrent Neural Networks (RNNs) is a very dynamic and robust sequential model and can learn long-term dependencies. According to recent research, it shows that by using normalization of the intermediate result of the neural network convergence rate of the network is improved. In this paper, the author shows how batch normalization is used in RNN. This model is applied to both speech reorganization and language modeling tasks [Laurent et al. 2016]. During the training of neural network parameters of each layer are change as the parameter of the previous layer is changed. In this paper, the author uses the concept of internal covariant shift and solves the problem by normalizing the layer input. Made use of an ensemble Network, the author tries to improve on the bestpublished Results on ImageNet classification [Kang et al. 2017]. A DNN based spectralspatial method is used to develop a hyperspectral Image classification. Every layer of the deep network uses spectral reduction as pre-processing and BN [Abbasi et al. 2019].

The idea of doing normalization before fusing classification scores of different levels that do not contain vectors. Experiment results are it has been shown that applied technique advances the learning process Better convergence and training for better neural networks the indoor RGBD provides a better understanding of visuals [Hayat et al. 2017]. Some standard technologies, as well as a model, are used for flood prediction are compared in this paper. The author provides a brief overview that the accuracy of every model depends on the different parameters [Dubey and Katarya 2020]. XGBoost and KNN are also providing better results for the data of flood classification. In this paper for flood, prediction the author uses various parameters such as elevation, the slope angle of that geographical area, and distance from the stream network. Among both of the techniques, XGBoost provides more accuracy [El-Magd et al. 2021].

PSO and Firefly have better results for optimizing weights for flash flood prediction [Khan et al. 2020]. Nowadays it is possible to forecast the occurrence of flash floods with the help of ML algorithms at the initial stage and is very helpful in minimizing the casualties. In various researches approaches for flood, detection the author uses a very small size dataset. So the accuracy of the model is very less. In many papers, authors were used ANN, but the layers are not so much dense. So the resultant accuracy is less. 
Table 1. Comparison table various for flood prediction approaches

\begin{tabular}{|c|c|c|c|c|}
\hline Paper & Dataset & Algorithm & Description & Limitations \\
\hline $\begin{array}{l}\text { Pyayt et } \\
\text { al., } 2011\end{array}$ & $\begin{array}{l}\text { Hyperspectral } \\
\text { Images }\end{array}$ & $\begin{array}{c}\text { Convolutional } \\
\text { Neural Network } \\
\text { and Principal } \\
\text { Components } \\
\text { Analysis(PCA) }\end{array}$ & $\begin{array}{l}\text { For HSI classification } \\
\text { this paper focuses on } \\
\text { the PBCNN } \\
\text { framework. PCA is } \\
\text { used to reduce } \\
\text { spectral } \\
\text { dimensionality. }\end{array}$ & $\begin{array}{l}\text { An increase in the } \\
\text { number of images } \\
\text { in the dataset can } \\
\text { provide better } \\
\text { accuracy }\end{array}$ \\
\hline $\begin{array}{l}\text { Kim et al. } \\
2016\end{array}$ & $\begin{array}{c}\text { The dataset } \\
\text { includes the } \\
\text { presence of } 201 \\
\text { floods from Iran's } \\
\text { Mazandaran } \\
\text { province. }\end{array}$ & $\begin{array}{l}\text { Various } \\
\text { Ensemble } \\
\text { models were } \\
\text { used with eight } \\
\text { different ML } \\
\text { models }\end{array}$ & $\begin{array}{l}\text { Various Ensemble } \\
\text { models (EM) were } \\
\text { used with eight } \\
\text { different ML models } \\
\text { EMmedian resulted in } \\
\text { the highest accuracy }\end{array}$ & $\begin{array}{l}\text { Work performed on } \\
\text { a limited data set, } \\
\text { which leads to low } \\
\text { model accuracy. }\end{array}$ \\
\hline $\begin{array}{l}\text { Saeed et } \\
\text { al. } 2018\end{array}$ & $\begin{array}{c}\text { In this paper, } \\
\text { multiple sensor } \\
\text { values are named } \\
\text { as Humidity, } \\
\text { Water Flow, } \\
\text { Water Level. }\end{array}$ & $\begin{array}{c}\text { CNN and Big } \\
\text { data }\end{array}$ & $\begin{array}{l}\text { This study IoT data IS } \\
\text { manage using HDFS } \\
\text { map-reduce is used. } \\
\text { For classification } \\
\text { Deep neural network } \\
\text { is developed. }\end{array}$ & $\begin{array}{l}\text { In the future we get } \\
\text { more enhanced } \\
\text { results with more } \\
\text { sensors and less cost, } \\
\text { it is possible to } \\
\text { detect floods using } \\
\text { the IoT. }\end{array}$ \\
\hline $\begin{array}{l}\text { Alquisola } \\
\text { et al. } 2018\end{array}$ & $\begin{array}{l}\text { Hydrodynamic } \\
\text { parameters } \\
\text { collected from } \\
\text { local stations and } \\
16 \text { different } \\
\text { datasets were } \\
\text { collected from } \\
\text { local meteorology }\end{array}$ & $\begin{array}{c}\text { ANN-based } \\
\text { prediction model }\end{array}$ & $\begin{array}{l}\text { An ANN-based after- } \\
\text { runner forecast model } \\
\text { is developed to } \\
\text { predict future } \\
\text { volatility. That leads } \\
\text { to Time (eg, } 24 \text { hours) }\end{array}$ & $\begin{array}{l}\text { An increase in the } \\
\text { number of neurons in } \\
\text { the hidden layers and } \\
\text { more thunderstorm } \\
\text { events to be } \\
\text { considered in the } \\
\text { forecast model }\end{array}$ \\
\hline $\begin{array}{l}\text { Anbarasan } \\
\text { et al. } 2020\end{array}$ & $\begin{array}{c}\text { Input data } \\
\text { meteorological } \\
\text { and Gloves. } \\
\text { Hydrological data } \\
\text { obtained }\end{array}$ & $\begin{array}{l}\text { Integrated Large } \\
\text { and crowdsource } \\
\text { data using ML } \\
\text { techniques }\end{array}$ & $\begin{array}{l}\text { The author uses } \\
\text { various ML } \\
\text { techniques, among } \\
\text { them ANN, SVM and } \\
\text { RF provides better } \\
\text { classification } \\
\text { accuracy }\end{array}$ & $\begin{array}{l}\text { Improve the } \\
\text { limitation is include } \\
\text { Incorporating the } \\
\text { Internet of Things } \\
\text { (IoT) into the real } \\
\text { measure } \\
\text { Meteorological data }\end{array}$ \\
\hline & A solar panel with & A nonlinear & The approach of this & The resulting \\
\hline
\end{tabular}




\begin{tabular}{|c|c|c|c|c|}
\hline $\begin{array}{l}\text { Puttinaov } \\
\text { arat et al. } \\
2020 \text {. }\end{array}$ & $\begin{array}{l}\text { a microcontroller, } \\
\text { GSM module, and } \\
\text { ultrasonic sensor } \\
\text { are used. }\end{array}$ & $\begin{array}{l}\text { autoregressive } \\
\text { network with } \\
\text { feed-forward } \\
\text { ANN with the } \\
\text { Backpropagation } \\
\text { technique is } \\
\text { used. }\end{array}$ & $\begin{array}{l}\text { paper is categorized } \\
\text { into three parts, In this } \\
\text { main data collection is } \\
\text { done. then describe } \\
\text { the hardware } \\
\text { architecture and last is } \\
\text { the architecture of the } \\
\text { ANN model and } \\
\text { predictions. }\end{array}$ & $\begin{array}{c}\text { estimated flood } \\
\text { water level matches } \\
\text { well with the } \\
\text { observed water } \\
\text { levels for both the } \\
\text { NARX and NAR } \\
\text { models. The NARX } \\
\text { model provides } \\
\text { better prediction } \\
\text { performance than the } \\
\text { NAR model. }\end{array}$ \\
\hline $\begin{array}{r}\text { Mousavi } \\
\text { et al. } 2021\end{array}$ & NASA Earth Data & $\begin{array}{c}\text { Convolutional } \\
\text { Neural Network }\end{array}$ & $\begin{array}{l}\text { The experiment was } \\
\text { done by using the } \\
\text { Caffe framework and } \\
\text { for CNN ResNet } 20 \text {, } \\
\text { ResNet50, } \\
\text { ResNet152, and } \\
\text { Inception models } \\
\text { were used }\end{array}$ & $\begin{array}{l}\text { Efficient algorithms } \\
\text { are needed to } \\
\text { improve the training } \\
\text { time of CNN's. }\end{array}$ \\
\hline $\begin{array}{l}\text { Khan et al. } \\
2020\end{array}$ & $\begin{array}{c}\text { Multiple sensor } \\
\text { data and Satellite } \\
\text { images }\end{array}$ & $\begin{array}{c}\text { AI and ML- } \\
\text { based algorithms }\end{array}$ & $\begin{array}{l}\text { All major AI-based } \\
\text { algorithms, sensor- } \\
\text { based and modeling } \\
\text { based models for flash } \\
\text { flood detection are } \\
\text { discussed }\end{array}$ & $\begin{array}{l}\text { The forecast is for } \\
\text { better performance to } \\
\text { optimize flood firefly } \\
\text { and PSO is used in } \\
\text { the future. }\end{array}$ \\
\hline $\begin{array}{l}\text { Liu et al. } \\
2021\end{array}$ & $\begin{array}{l}11 \text { flood condition } \\
\text { factors from the } \\
\text { various standard } \\
\text { dataset }\end{array}$ & SVM & $\begin{array}{l}\text { The author introduced } \\
\text { the FSCI to measure } \\
\text { the vulnerability of } \\
\text { floods. this is } \\
\text { achieved by using } \\
\text { SVM }\end{array}$ & $\begin{array}{l}\text { Future studies should } \\
\text { consider more } \\
\text { comprehensive } \\
\text { indicator systems, } \\
\text { climate change } \\
\text { factors, and higher } \\
\text { quality flood points. }\end{array}$ \\
\hline $\begin{array}{l}\text { Charbuty } \\
\text { and } \\
\text { Abdulaze } \\
\text { ez et al. } \\
2021 \text {. }\end{array}$ & $\begin{array}{c}\text { Environmental } \\
\text { sensor data }\end{array}$ & $\begin{array}{c}\text { Decision tree } \\
\text { classifiers }\end{array}$ & $\begin{array}{l}\text { In this paper, the } \\
\text { author provides a } \\
\text { detailed description of } \\
\text { decision trees for } \\
\text { classification. }\end{array}$ & $\begin{array}{c}\text { The algorithm } \\
\text { provides better } \\
\text { accuracy if more } \\
\text { data instances are } \\
\text { used. }\end{array}$ \\
\hline
\end{tabular}


In the above survey, we provide an overview of the various AI, ML, and DL-based approaches for flood prediction as well as detection. The experiments are performed on various datasets that contain environmental parameters and satellite images for prediction.

\section{Preliminaries for Flood disaster detection system}

Worldwide all the countries are concern about natural disasters. There are two types of disasters, natural disasters like tsunamis, forest-fire, lightning, flood, earthquake, and also landslide, and man-made disasters like leakage in gas production, leakage in an oil pipeline, industrial explosion, and man-made disaster can be controlled or saved by a man.

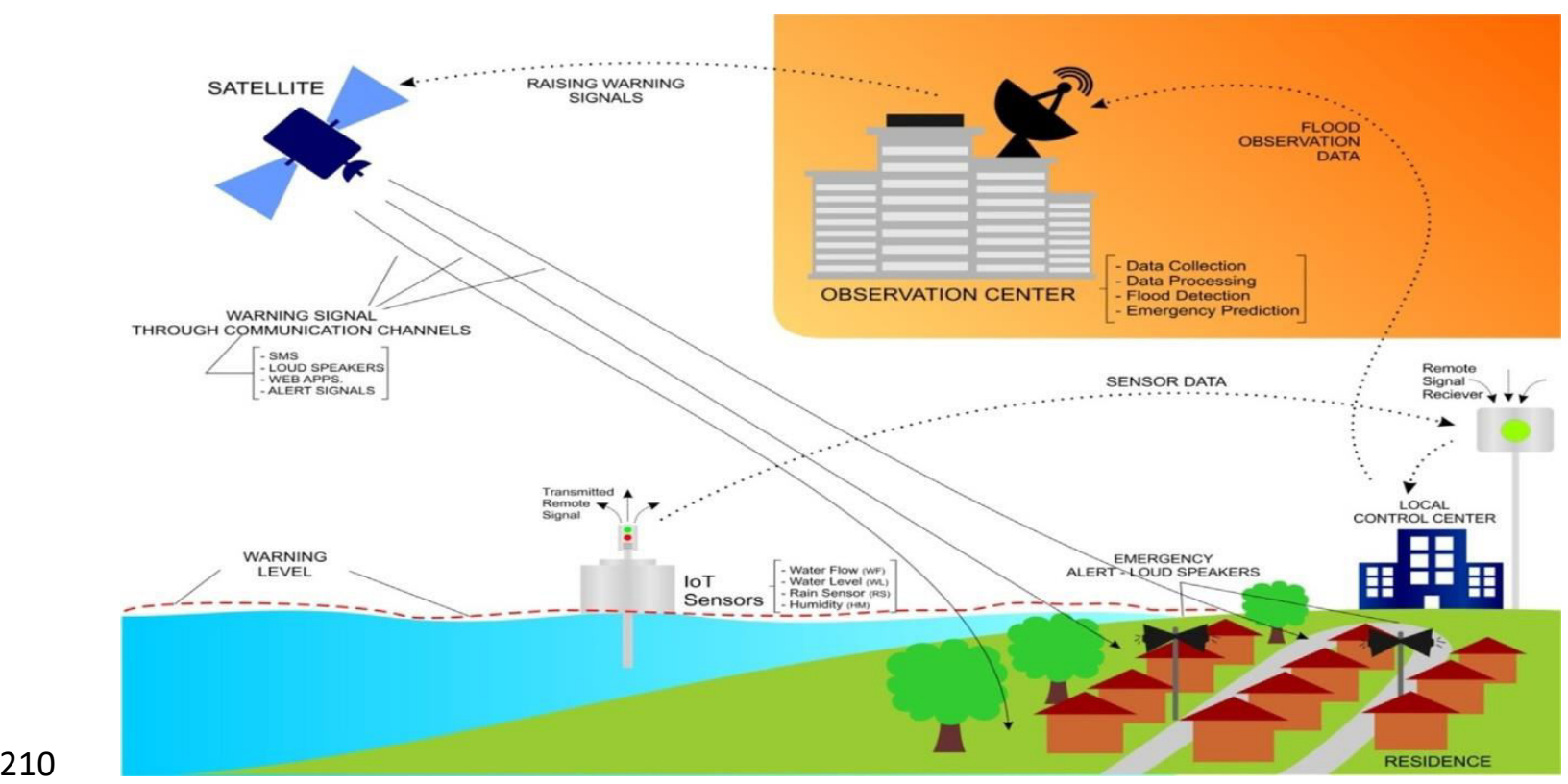

Fig 1: Working of Flood Disaster Early Warning System [Saeed et al. 2018] framework development is recommended.

- All research is done on the basic ML and AI-based algorithm which provides less accuracy. In the case of Deep neural networks, there is a need to add a more dense hidden layer for providing better accuracy.

- Dataset considered in various research is very small that causes the lower performance of the algorithm. 
212 In the above figure 1 give an overview that how with help of current technologies an 213 effective Flood prediction system is designed. The proposed approach extracts various 214 features that are used to predict flooding from the climate using different types of sensors and predict the probability of flooding with certain prediction algorithms. But in the case of natural calamities, it is possible we only create an early prediction system to predict the occurrence of the disaster so that we can take some precautions. Among all-natural disasters, flood very commonly occurs in natural disasters. In this paper, we have a focus on flood disaster detection systems. People will be prevented to face such a disaster from early detection of disaster. Here an overvirw of verious ML algorithms is provides.

\subsection{Support Vector Machine}

SVM is a supervised learning algorithm that may be used to tackle regression and different data instances in SVM. Here we focus on identifying the hyperplane with the largest margin, or distance between data points from both classes. The sets to differentiate are usually not linearly separable in that space, even though the original problem is described in a finite-dimensional space. Maximizing distance helps classify data points. Also, it allows data points to be assigned to different classes. [Yan et al. 2017].

In addition, the number of features determines the hyper plane's dimension. Because supervised learning is impossible when data is unlabeled, an unsupervised learning method is required, in which data is spontaneously grouped into groups and new data is mapped to these classes. In this approach, for generating a flood sensitivity map SVM model is used based on 11 factors of flood conditions [Liu et al. 2021]. Even though the initial problem is expressed in a finite-dimensional space, the sets to differentiate are often not linearly separable in that space.

\subsection{Decision Tree}

The DT is one of the regularly used machine learning algorithms. It is the most popular and powerful tool for data prediction and classification. DT is a kind of supervised learning algorithm. Where each data instance belongs to a specific class. This structure is a tree-like representation of the features of a dataset, which are represented by the branches and the leaf nodes. It shows the various decisions and solutions that can be derived from the dataset. This algorithm is used to build a model to identify the value of the target variable. For which a tree-like structure is used where the leaf node represents the class label for the corresponding problem. The internal node attributes are represented by that tree. [Somvanshi et al. 2016]. 
248 When we talk about flood prediction systems then DT is very useful and commonly used 249 The classification algorithm is used for predicting the likelihood of floods based on the 250 collected data. It also classifies the data according to its classification. [Charbuty and 251 Abdulazeez et al. 2021].

\section{3.3 Artificial Neural Network}

An ANN is an ML model that draws on the principles of biological neurons. It learns and uses information flow to make predictions. ANN can be classified into a feed-forward (static) network and feed backward (Recurring) network. A feed-forward neural network implementation performs non-linear functions of provided input. It is a nonlinear function of neurons that is determined by nonlinear, bias functions and weight [Dutt et al. 2018].

ANN is the most generally used data processing technique. ANN has been applied in most studies with significant emphasis on prediction. The author was used ANN are used as a base model. Feedforward fully connected neural network is the very first neural network. This consists of several layers of neurons (nodes) connected. These layers are named the Output layer, Hidden layer, and Input layer. direct edges connect neurons in different layers, with each edge assigned some weight that is updated during the training of our network [Abiodun et al. 2019]. A fully connected feed-forward ANN is used here to give early warning for prediction and to communicate to the target users. In the neural network architecture, various activation functions are used in neural network architecture to activate neurons ReLU is used [Subeesh et al. 2018].

\subsection{Deep neural network}

Since the proposal of fast learning algorithms for deep belief networks, deep learning techniques have attracted ever-increasing research interest due to their inherent ability to overcome the shortcomings of traditional algorithms relying on hand-designed features. The basic unit of the neural network is called neurons/ perceptrons. So how neural network does is work? Multiple inputs are provided to the neurons named $\mathrm{x} 1$, $\mathrm{x} 2$, etc. it depends on the number of input data instances and produces output depends upon the output classes [Kwasigroch et al. 2019].

Not only can DNNs solve a task according to an algorithm, but they can also use their experience to predict its solution. [Liu et al. 2017]. DNN is a distributed system that works seamlessly in multiple layers. This is beneficial when you need to use it to replace human labor without compromising on its efficiency. 


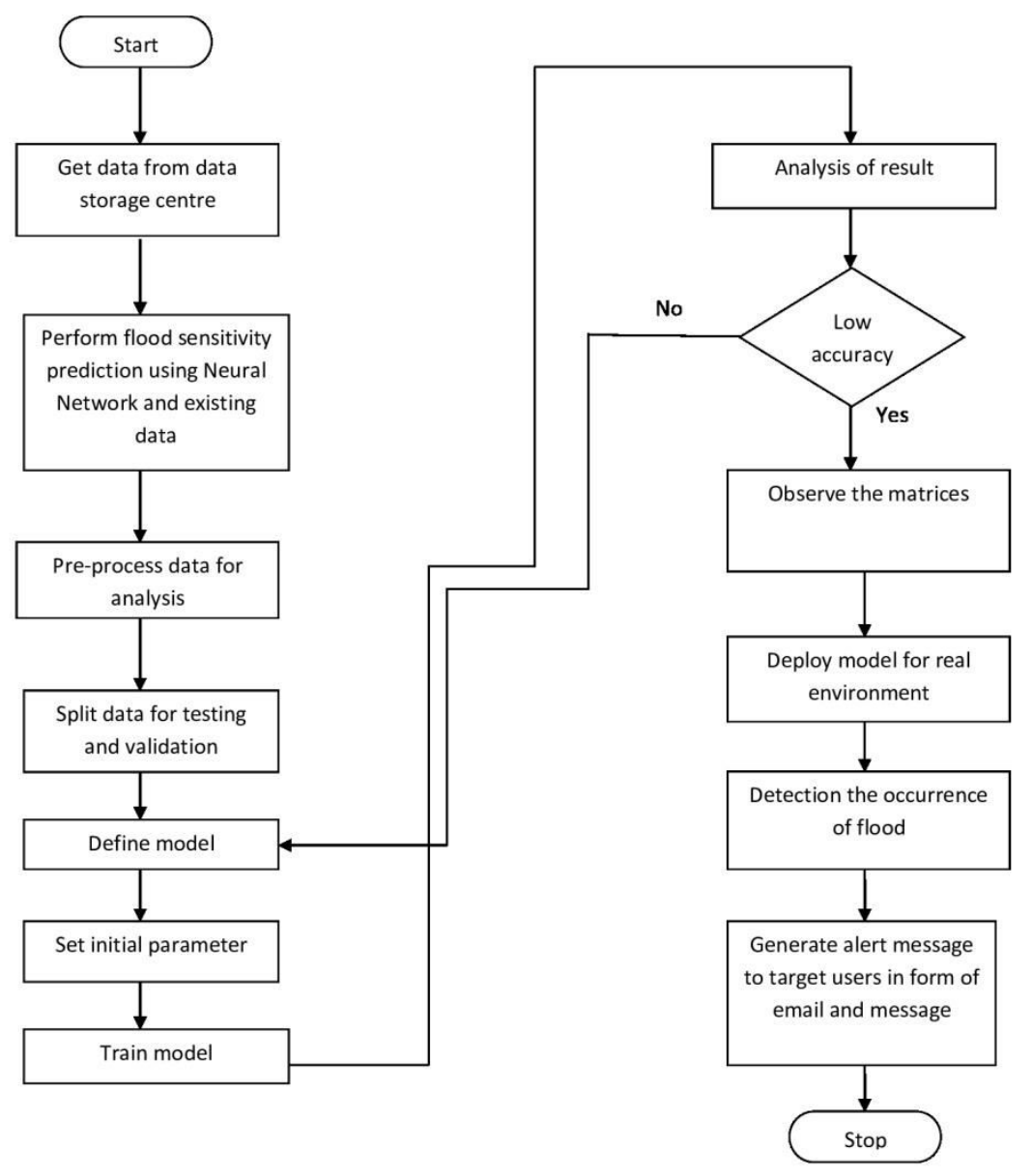

Fig 2: Represent Flow Chart of the proposed model

285 In the above figure 2, we present a brief overview of the working of the flood detection 286 system. We demonstrate the working of our proposed methodology. The process is 287 started from collect data from the data storage center then we pre-process the initial data. 288 Some techniques like ANN, DNN, and Batch Normalization are used for the 289 classification of data. Our data is classified into three classes named 'LOW', 290 MODERATE' and 'HIGH'. When real-time data is feed to the model then it will 291 respond. Initially, the threshold value of each attribute is set then when the flood is cross 292 its severity level then it sends the alert message regarding the flood to the high authorities 
293 to take appropriate action. In this model, we also send the alert message to the public 294 community live near the water bodies.

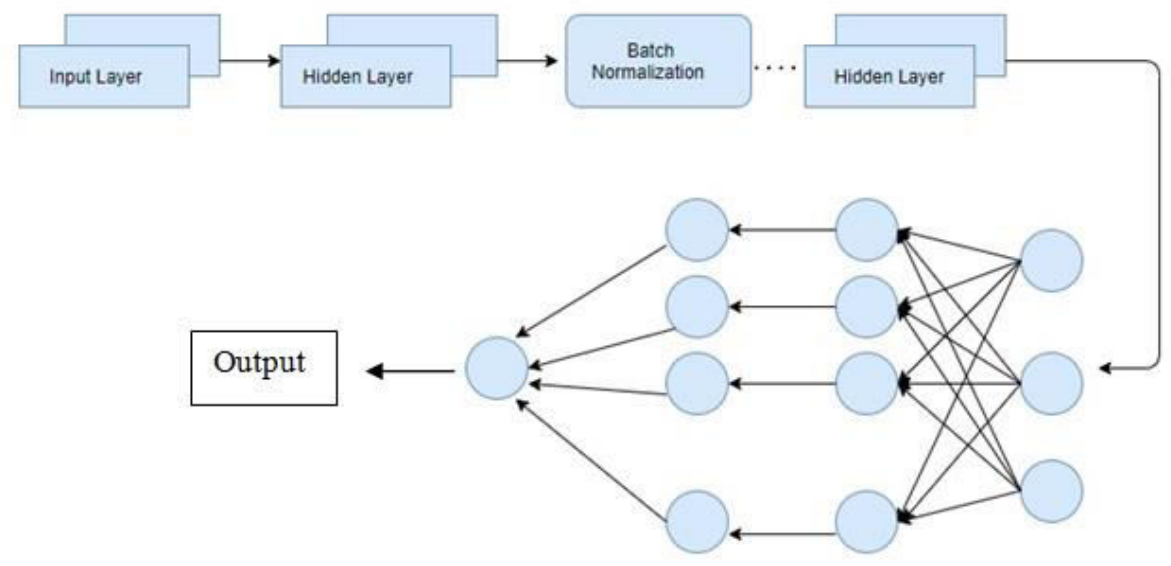

299 In above figure 3, we explain the basic structure of our proposed BNDNN model for 300 flood prediction based on the severity of the rain. The first layer of our model is the input 301 layer. The number of neurons in the input layer is dependent on the data instances. The 302 next layer of our model is the Hidden layer. The normalization technique is applied 303 between the output of the first hidden layer and the input to the next hidden layer. Batch 304 Normalization normalizes the input data to the activation function of each layer by using 305 the small mini-batches. In the output layer, we use a single neuron and predict the 306 possibility of flooding outside based on rainfall.

307 In our model, to introduce non-linearity into the model ReLU activation function is used. 308 For classification, the softmax activation function is used to obtain the probability of 309 being between 0 and 1 . The equation of ReLU and softmax function is given below.

310 In neural networks, the Cross-entropy loss function is used while training our network. 311 For prediction and classification, problem cross-entropy error is provide better results. It 312 predicts a class highest likelihood evaluation based on input. It is an alternative to the 313 cross-entropy loss function and the mean square error. The equation of the cross-entropy 314 loss function is given below.

$$
H_{Y^{\prime}}(Y)=-\sum_{i} y_{i}^{\prime} \log \left(y_{i}\right)
$$


317 In the above equation, $1 \mathrm{y}_{\mathrm{i}}$ is the predicted probability value for class $\mathrm{i}$, and $\mathrm{y}_{\mathrm{i}}$ ' is the true

318 probability for that class. $H_{Y^{\prime}}(Y)$ is the loss function. The error is calculated after the

319 first iteration is complete and it spreads back to the input layer in the previous layers and

320 computes the gradient. For the learning of neural networks, back-propagation techniques

321 are used. The approach here for optimizing the neural network is called gradient descent

322 for adjusts the weight. Weight is updated according to the formula below.

$$
\omega=\omega-\mu \nabla Q(\omega)
$$

324 In equation $2, Q$ is the phase of the shape or sometimes called the learning rate. $\omega$ is the 325 weight assign to neurons. This reduces further errors. In this method stochastic gradient 326 descent method is adopted, the method adopted is based on the calculation of cost 327 gradient. For all the training data during the iteration, this process is repeated. Then our 328 network is well learned to predict the outputs for the test inputs.

329 Batch Normalization normalizes the input data to the activation function of each layer by 330 using the small mini-batches. The batch has the standard normal distribution (with 331 standard deviation is 1 and mean is 0 ).

332 Let us consider mini-batch is $\mathrm{B}$, size of mini-batch is $\mathrm{M}$ (consider as entire data-set) i.e

$333 \quad B N_{\gamma, \beta}: x_{1 \ldots \ldots \ldots . m} \rightarrow y_{1 \ldots \ldots \ldots m}$

$334 \quad \mathrm{~B}=\left\{\mathrm{X}_{1}, \ldots \ldots \ldots \ldots \ldots \ldots \ldots \ldots \ldots \ldots, \mathrm{X}_{\mathrm{M}}\right\}$

335 The Batch Normalization is

336

$$
B N_{\gamma, \beta}: \quad x_{1 \ldots \ldots \ldots m} \rightarrow y_{1 \ldots \ldots \ldots m}
$$

337 After calculating variance and mean of small-batch data we normalize it

$$
\widehat{x}_{\imath} \leftarrow \frac{x_{i}-\mu_{B}}{\sqrt{\sigma_{B}^{2}-\varepsilon}}
$$

$\mu_{B}$ is sample mean

$\sigma_{B}^{2}$ is sample variance

342 Scale and shift is represented as follows 


$$
y_{i} \leftarrow \gamma \widehat{x}_{\imath}+\beta \equiv B N_{\gamma, \beta}\left(x_{i}\right)
$$

$344 \quad$ Where $\gamma$ and $\beta$ need to be calculated

345 When the mean and variance are calculated over the entire training dataset that 346 normalization increases the speed of the network. While normalizing each input of layer 347 will lose the information transferred from the previous layer.

348 In Batch Normalization, if we use to normalize the entire dataset in each training set, it is 349 not useful when we use a stochastic optimizer. So another method of normalization is 350 used. While using stochastic gradient training, in this approach each mini-batch is 351 calculated mean and variance of each activation. In this, the data used for normalization 352 can be fully used in gradient backpropagation. Consider the mini-batch B with size $\mathrm{m}$, in 353 this, we applied normalization to each activation function.

$$
\mu_{B} \leftarrow \frac{1}{m} \sum_{i=1}^{m} x_{i}
$$

$$
\sigma_{B}^{2} \leftarrow \frac{1}{m} \sum_{i=1}^{m}\left(x_{i}-\mu_{B}\right)^{2}
$$

This approach is used to improve the performance, and speed of ANN. It normalizes the output of the input layer by re-scaling and re-centering. This allows us to be less careful about higher learning rates and initialization. In $\mathrm{BN}$ each layer has some impact according to random distribution. The input is changed during the training of the network due to the randomness of data. The effect of this randomness on the input to the internal layer is defined as the internal covariant shift. To resolve the issue of the internal covariant shift we have to standardize every input layer. It is very time-consuming and less effective. So to solve this problem batch normalization is introduced to reduce the internal covariant shift. 


\section{5. Result and Discussion}

\section{$373 \quad 5.1$ Data collection}

374 The main thing to design a flood natural disaster prediction system is to need a huge 375 dataset to process and generate the result; data is a very important part of any prediction 376 model. Data can be used in any format such as images, videos, etc. For forecasting, data 377 collected from any source sensor, satellite, the video camera is used. A large area of India 378 is a flood-prone region as most of the human communities are located in nearby rivers. 379 Therefore, they face floods for the maximum time during the rainy season. India is very 380 suitable for flood detection. Following this, many of the states and union territories of 381 India were facing major floods.

382 By doing this, it was found that Bihar has the highest risk of floods in the country. 383 According to International Water Management Institute (IWMI), every year 73 percent of 384 the total surface gets submerged. In Orissa to the frequency of flood events was very 385 high. So we include two main states of India, Bihar, and Orissa for our system. Since 386 Bihar and Orissa are both very large states, districts in these two states is 38 and 30 387 respectively, we select the ten most flood-prone cities from each state. For our prediction 388 system, we use a standard dataset that includes parameters like minimum temperature, 389 maximum temperature, precipitation; vapor pressure along with this parameter year, the 390 month is also included in the dataset. We have considered over fifteen years between 391 January to December.

\subsection{Confusion matrix}

393 The confusion matrix is a type of binary classifier that can be any size depends upon the 394 number of different classes/ labels. Here the size of the matrix is (3X3) as we divide our 395 data into three classes Low, Moderate and High. This is a table with four different 396 combinations of estimated and actual values. These combinations are TP, FP, TN, and 397 FN.

$\mathrm{TP}=$ true positive

$399 \quad \mathrm{FP}=$ false positive

$400 \mathrm{TN}=$ true negative

$401 \quad \mathrm{FN}=$ false negative

402 Where TP and TN denote the number of samples correctly classified the severity of the 403 flood respectively. FP and FN denote the number of samples that have been incorrectly 404 classified based on the severity of flood events.

405 In our paper, all four values of TP, FP, TN, and FN are extremely useful for calculating 406 the various parameters named Precision, Recall, F1-score, and accuracy. 


\subsubsection{Precision}

409 Precision is calculated as the fraction of correctly identifies instances among all instances 410 which are identified as positive, which means how many instances are positive. For better 411 results precision should be high. Precision is calculated using the following formula

$$
\text { Precision }=\frac{T P}{T P+F P}
$$

413 High precision requires a low FN rate and a low FP rate.

\section{$414 \quad 5.2 .2$ Recall}

415 The Recall is a metric that measures the number of true positive predictions made from 416 all positive predictions that could have been made. The percentage of the total positive is 417 estimated to be positive. It is similar to TPR (true positive rate). For unbalanced learning, 418 recall is typically used to measure minority class coverage. In our model or algorithm, we 419 focus on the high recall value. The recall is calculated using the following formula

$$
\text { Recall }=\frac{T P}{T P+F N}
$$

421 High precision requires a low FN rate and a low FP rate.

\section{$422 \quad$ 5.2.3 F1-score}

423 F1-score is a matrix; the accuracy and recall of both the values are needed to calculate the

424 F1-score. It is a harmonic average of recall and precision wherein the best-case F1 value 425 is 1 and in the worst case, the F1 value is 0. To calculate F1-score following formula is 426 used

$$
\text { F1-score }=2 * \frac{\text { Precision } * \text { Recall }}{\text { Precision }+ \text { Reall }}
$$

\section{$428 \quad 5.2 .4$ Accuracy}

429 Based on the confusion, matrix, for calculating the accuracy of the ratio of the correctly 430 predicting instances to the total instances in the dataset. The formula to calculate 431 accuracy is given below

$$
\text { Accuracy }=\frac{T P+T N}{T P+F P+T N+F N}
$$


436 In this section, we have shone the accuracy, precision, F-score, and Recall of our 437 proposed model and some other machine learning algorithms and compare their results. 438 For this, we collect data from data storage then pre-processed the data to feed to our network. Here we have train five models and machine learning algorithms named SVM, 440 DT, ANN, DNN, and our proposed model DNN with Batch Normalization (BNDNN). 441 We divide the dataset into training, validation, and testing categories. From the dataset $44270 \%$ of data is used for training the model $15 \%$ is used for validation and $15 \%$ is used for 443 testing. According to the severity of the flood, data is divided into three classes named as 444 Low, Moderate, and High chance of flood.

445 Table 1 Cooperative tabulation of Proposed Model (BNDNN), SVM, DT, 446 ANN and DNN

\begin{tabular}{|c|c|c|c|c|}
\hline Model/ Algorithm & & Precision & Recall & F1-score \\
\hline \multirow[t]{4}{*}{ SVM } & Low & 90 & 70 & 85 \\
\hline & Moderate & 76 & 87 & 83 \\
\hline & High & 77 & 90 & 87 \\
\hline & Accuracy & \multicolumn{3}{|c|}{83} \\
\hline \multirow[t]{4}{*}{ DT } & Low & 93 & 76 & 82 \\
\hline & Moderate & 75 & 92 & 80 \\
\hline & High & 73 & 87 & 83 \\
\hline & \begin{tabular}{|l|} 
Accuracy \\
\end{tabular} & \multicolumn{3}{|c|}{80} \\
\hline \multirow[t]{4}{*}{$\mathbf{A N N}$} & Low & 92 & 98 & 79 \\
\hline & Moderate & 70 & 94 & 82 \\
\hline & High & 75 & 89 & 85 \\
\hline & Accuracy & \multicolumn{3}{|c|}{82} \\
\hline \multirow{4}{*}{ DNN } & Low & 95 & 72 & 83 \\
\hline & Moderate & 94 & 97 & 87 \\
\hline & High & 78 & 82 & 91 \\
\hline & Accuracy & \multicolumn{3}{|c|}{84} \\
\hline \multirow{3}{*}{$\begin{array}{c}\text { Proposed Model } \\
\text { (BNDNN) }\end{array}$} & Low & 98 & 80 & 89 \\
\hline & Moderate & 81 & 99 & 92 \\
\hline & High & 84 & 95 & 94 \\
\hline
\end{tabular}

448 In the above table 1, we have compared the four existing models and ML algorithms such 449 as ANN, DNN, SVM, and DT with our proposed model. The comparison is done based 450 on the parameter like F1-score, recall, precision, and accuracy of the models. Here we 451 calculate the F1-score, recall, and precision for all the three classes of every technique 452 and at the end, we calculate the accuracy of the model and algorithm. Based on the above 
453 comparison, we say that the proposed model in this paper Batch Normalization with 454 Neural Network (BNDNN) is performing better. We get higher accuracy with our model.

\section{$455 \quad 5.5$ Performance Analysis}

456 Here the BNDNN is performed better in comparison to SVM, DT, ANN, and DNN on 457 the dataset collected from the data storage center. Based on the above comparison, we say 458 that the proposed model in this paper Batch Normalization with Neural Network 459 (BNDNN) is performing better. We get higher accuracy with our model. In this section, 460 we discuss the graphical representation and identify that which machine learning 461 algorithms such as ANN, SVN, DT, DNN, and our proposed model BNDNN are 462 predicting the severity of the flood. Before the result, we provide a brief overview of 463 performance metrics and parameters such as precision, recall, F1-score, and accuracy 464 used for the analysis of the result.

465

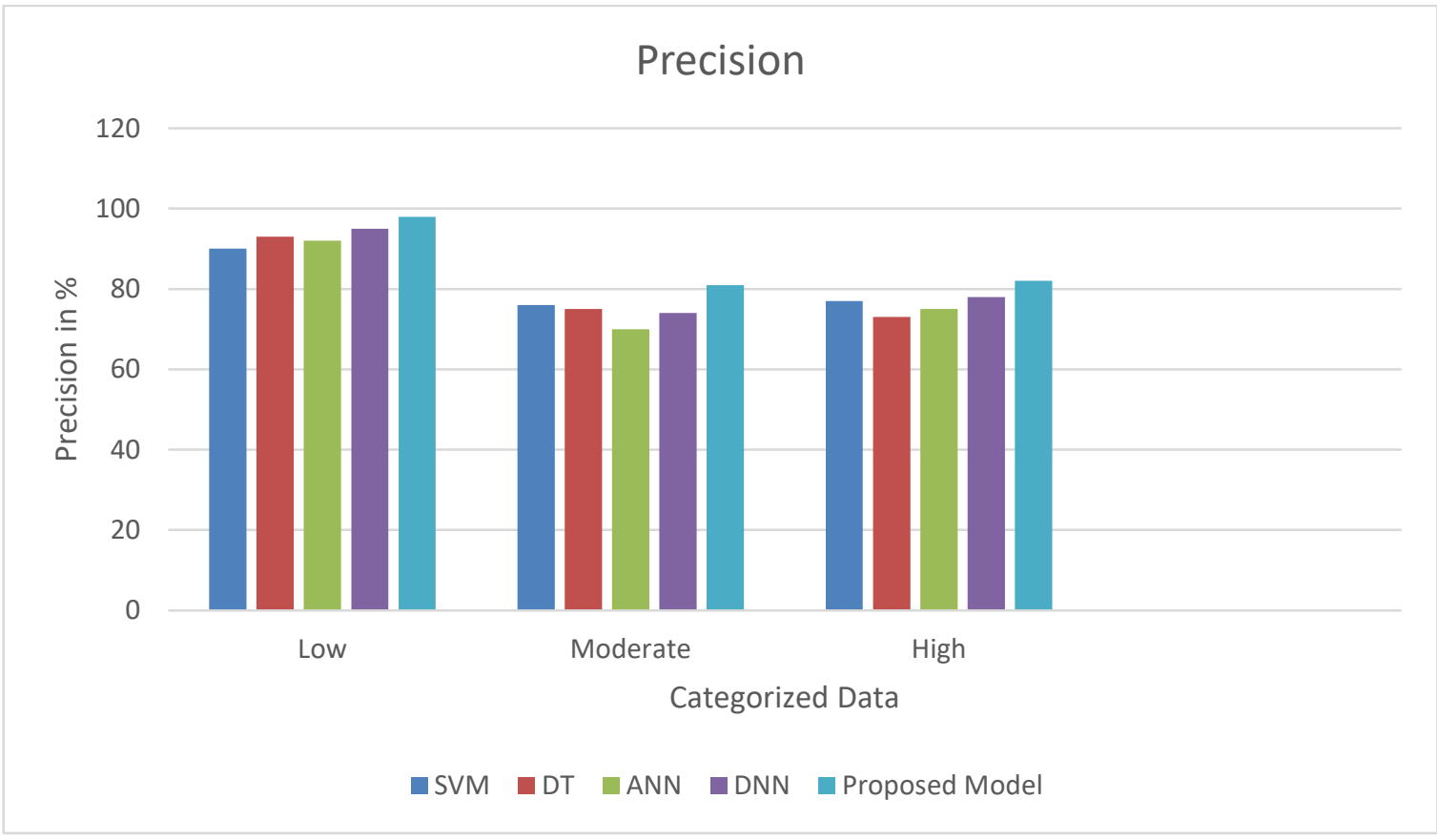

467 Fig. 4 Graph between Categorized data and Precision of SVM, DT, ANN, DNN, and 468 Proposed Model

469 In the above figure 4, a graphical representation for the precision of various machine 470 learning algorithms and our proposed model. The performance of each model varies in 471 each data class but our proposed model BNDNN performs better in each case. 


\section{Recall}

120

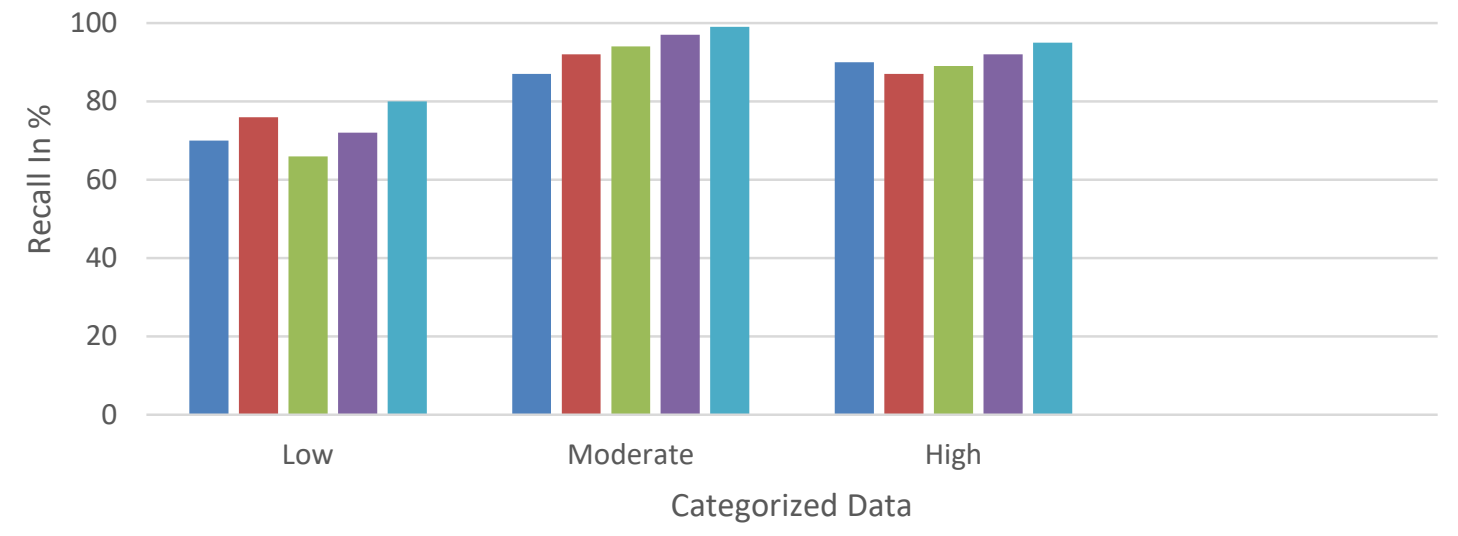

473 Fig. 5 Graph between Categorized data and Recall of SVM, DT, ANN, DNN, and 474 Proposed Model

475 In the above figure 5, we represent the graphical comparison for the precision of various 476 machine learning algorithms and our proposed model. Our proposed model BNDNN 477 provides better prediction for all data classes.

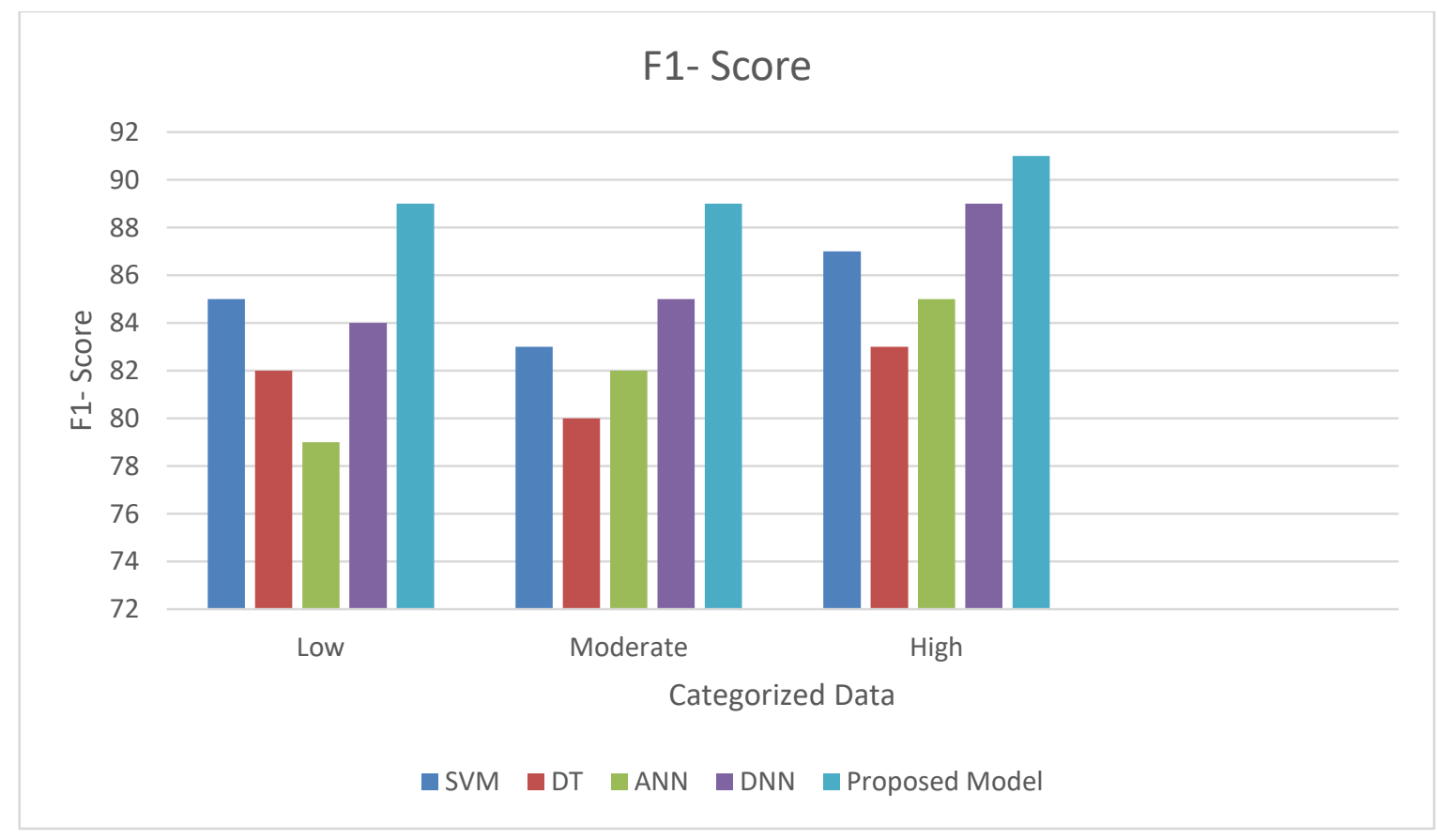

479 Fig. 6 Graph between Categorized data and F1-score of SVM, DT, ANN, DNN, and 480 Proposed Model 
In the above figure 6 , the graphical representation for the F1-score of various machine learning algorithms and our proposed model is provided. Other machine learning models are performing poorly in each class. Our proposed model BNDNN provides far better results than the rest of the model.

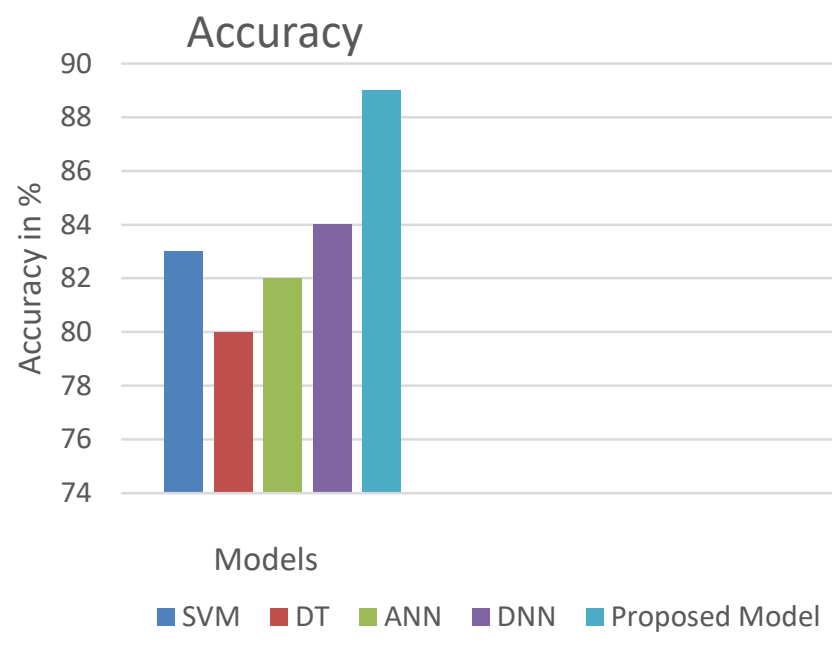

486 Fig. 7 Graph between Model Accuracy score of SVM, DT, ANN, DNN, and Proposed Model

In the above figure 7, The graphical representation of the accuracy of various machine learning models and our BNDNN proposed model is provided. As a result, our proposed model provides $89 \%$ accuracy which is the highest of all accuracy. DNN with $84 \%$ accuracy outperforms SVM with $83 \%$ accuracy. ANN provides $82 \%$ accuracy while DT provides very low $80 \%$ accuracy.

It is visible after analyzing the result based on various parameters that our proposed model (BNDNN) provides better accuracy than other algorithms. The rest of the parameters such as precision, recall, and F1-score are also providing better resultant value as compared to other algorithms. For an efficient model or algorithm, the value of precision and recall should be high. The value of the F1-score depends on the precision and recall value, so higher precision and recall give better results.

\section{Conclusion and Future Scope}

500 We cannot control human-made disasters because they are unpredictable. To control, we need human awareness and seriousness in human labor disasters. But in case of a natural disaster like flood, fire, cyclone, etc., we can warn people about their occurrence with previous experience and generate a vigilance system with the help of currently available technologies, so that we save diversity can. In this paper, we used a neural network for the prediction of the flood. In this paper, we present a new approach with the vast 
capability of recent technologies like DNN combine with batch normalization for the implementation of an effective flood detection system that can combat flood natural disasters.

Our proposed model works on the concept of batch normalization technique. We compare our proposed model with SVM, DT, ANN, and DNN models. We train our model on the standard dataset. We compare our model based on the parameter like accuracy, precision, recall, F1-score. The accuracy of SVM is $83 \%$, DT is $80 \%$, ANN is $82 \%$ and DNN is $84 \%$ respectively. Whereas our proposed model (BNDNN) stores $89 \%$ accuracy higher than all other approaches. After comparison on the above parameter, we can conclude that our proposed model gives more accuracy than other models used for prediction. In the future, if we use more environmental parameters the there is a chance to

\section{Reference}

527 Hirabayashi Y, Mahendran R, Koirala S, Konoshima L, Yamazaki D, Watanabe S, Kim H, Kanae 528 S (2013) Global flood risk under climate change. Nature Climate Change 3:816-821. doi: $52910.1038 /$ nclimate1911

530 Dottori, F., Salamon, P., Bianchi, A., Alfieri, L., Hirpa, F. A., \& Feyen, L. (2016). Development 531 and evaluation of a framework for global flood hazard mapping. Advances in Water Resources, 532 94, 87-102. https://doi.org/10.1016/j.advwatres.2016.05.002

533 Mosavi, A., Ozturk, P., \& Chau, K. W. (2018). Flood prediction using machine learning models:

534 Literature review. Water (Switzerland), 10(11), 1-40. https://doi.org/10.3390/w10111536

535 Li, X., Yan, D., Wang, K., Weng, B., Qin, T., \& Liu, S. (2019). Flood risk assessment of global 536 watersheds based on multiple machine learning models. Water (Switzerland), 11(8), 1-18.

537 https://doi.org/10.3390/w11081654

538 Liu Q, Li Y, Yu M, Chiu L, Hao X, Duffy D, Yang C (2019) Daytime Rainy Cloud Detection and 539 Convective Precipitation Delineation Based on a Deep Neural Network Method Using GOES-16 540 ABI Images. Remote Sensing 11:2555. doi: 10.3390/rs11212555 
Ahmadisharaf E, Tajrishy M, Alamdari N (2015) Integrating flood hazard into site selection of 542 detention basins using spatial multi-criteria decision-making. Journal of Environmental Planning 543 and Management 59:1397-1417. doi: 10.1080/09640568.2015.1077104

544 Aazam M, Khan I, Alsaffar A, Huh E (2014) Cloud of Things: Integrating Internet of Things and 545 cloud computing and the issues involved. Proceedings of 2014 11th International Bhurban 546 Conference on Applied Sciences \& Technology (IBCAST) Islamabad, Pakistan, 14th - 18th 547 January, 2014. doi: 10.1109/ibcast.2014.6778179

548 Pyayt, A. L., Mokhov, I. I., Lang, B., Krzhizhanovskaya, V. V., \& Meijer, R. J. (2011). Machine 549 learning methods for environmental monitoring and flood protection. World Academy of Science, 550 Engineering and Technology, 78(6), 118-123. https://doi.org/10.5281/zenodo.1075060

551 Saeed F, Paul A, Rehman A, Hong W, Seo H (2018) IoT-Based Intelligent Modeling of Smart 552 Home Environment for Fire Prevention and Safety. Journal of Sensor and Actuator Networks 553 7:11. doi: 10.3390/jsan7010011

554 Ranit A, Durge P (2019) Flood Forecasting by Using Machine Learning. 2019 International 555 Conference on Communication and Electronics Systems (ICCES). doi: 556 10.1109/icces45898.2019.9002579

557 Ghose D (2018) Measuring Discharge Using Back-Propagation Neural Network: A Case Study 558 on Brahmani River Basin. Advances in Intelligent Systems and Computing 591-598. doi: 559 10.1007/978-981-10-7566-7_59

560 Anbarasan M, Muthu B, Sivaparthipan C, Sundarasekar R, Kadry S, Krishnamoorthy S, Samuel 561 R. D, Dasel A (2020) Detection of flood disaster system based on IoT, big data and convolutional 562 deep neural network. Computer Communications 150:150-157. doi: 563 10.1016/j.comcom.2019.11.022

564 Mitra P, Ray R, Chatterjee R, Basu R, Saha P, Raha S, Barman R, Patra S, Biswas S, Saha S 565 (2016) Flood forecasting using Internet of things and artificial neural networks. 2016 IEEE 7th 566 Annual Information Technology, Electronics and Mobile Communication Conference 567 (IEMCON). doi: 10.1109/iemcon.2016.7746363

568 Alquisola G, Coronel D, Reolope B, Roque J, Acula D (2018) Prediction and Visualization of the 569 Disaster Risks in the Philippines Using Discrete Wavelet Transform (DWT), Autoregressive 570 Integrated Moving Average (ARIMA), and Artificial Neural Network (ANN). 2018 3rd 571 International Conference on Computer and Communication Systems (ICCCS). doi: $57210.1109 /$ ccoms.2018.8463238

573 Puttinaovarat S, Horkaew P (2020) Flood Forecasting System Based on Integrated Big and 574 Crowdsource Data by Using Machine Learning Techniques. IEEE Access 8:5885-5905. doi: 575 10.1109/access.2019.2963819 
Bande S, Shete V (2017) Smart flood disaster prediction system using IoT \& neural networks. 2017 International Conference On Smart Technologies For Smart Nation (SmartTechCon). doi: 10.1109/smarttechcon.2017.8358367

Kim S, Matsumi Y, Pan S, Mase H (2016) A real-time forecast model using artificial neural network for after-runner storm surges on the Tottori coast, Japan. Ocean Engineering 122:44-53. doi: 10.1016/j.oceaneng.2016.06.017

Sahagun M, Dela Cruz J, Garcia R (2017) Wireless sensor nodes for flood forecasting using artificial neural network. 2017IEEE 9th International Conference on Humanoid, Nanotechnology, Information Technology, Communication and Control, Environment and Management (HNICEM). doi: 10.1109/hnicem.2017.8269462

Lee S, Kim J, Jung H, Lee M, Lee S (2017) Spatial prediction of flood susceptibility using random-forest and boosted-tree models in Seoul metropolitan city, Korea. Geomatics, Natural Hazards and Risk 8:1185-1203. doi: 10.1080/19475705.2017.1308971

Hosseiny H, Nazari F, Smith V, Nataraj C (2020) A Framework for Modeling Flood Depth Using a Hybrid of Hydraulics and Machine Learning. Scientific Reports. doi: 10.1038/s41598-020$65232-5$

Mousavi F, Yousefi S, Abghari H, Ghasemzadeh A (2021) Design of an IoT-based Flood Early Detection System using Machine Learning. 2021 26th International Computer Conference, Computer Society of Iran (CSICC). doi: 10.1109/csicc52343.2021.9420594

Keong K, Mustafa M, Mohammad A, Sulaiman M, Abdullah N (2016) Artificial neural network flood prediction for sungai isap residence. 2016 IEEE International Conference on Automatic Control and Intelligent Systems (I2CACIS). doi: 10.1109/i2cacis.2016.7885321

Shafizadeh-Moghadam H, Valavi R, Shahabi H, Chapi K, Shirzadi A (2018) Novel forecasting approaches using combination of machine learning and statistical models for flood susceptibility mapping. Journal of Environmental Management 217:1-11. doi: 10.1016/j.jenvman.2018.03.089

Al Nuaimi E, Al Neyadi H, Mohamed N, Al-Jaroodi J (2015) Applications of big data to smart cities. Journal of Internet Services and Applications. doi: 10.1186/s13174-015-0041-5

Chen L, Fei H, Xiao Y, He J, Li H (2017) Why batch normalization works? a buckling perspective. 2017 IEEE International Conference on Information and Automation (ICIA). doi: 10.1109/icinfa.2017.8079081

Duan J, Zhang R, Huang J, Zhu Q (2018) The Speed Improvement by Merging Batch Normalization into Previously Linear Layer in CNN. 2018 International Conference on Audio, Language and Image Processing (ICALIP). doi: 10.1109/icalip.2018.8455587

Laurent C, Pereyra G, Brakel P, Zhang Y, Bengio Y (2016) Batch normalized recurrent neural networks. 2016 IEEE International Conference on Acoustics, Speech and Signal Processing (ICASSP). doi: 10.1109/icassp.2016.7472159 
612 Kang D, Park M, Kim H, Kim D, Kim S, Son H, Lee S (2017) Room Temperature Control and 613 Fire Alarm/Suppression IoT Service Using MQTT on AWS. 2017 International Conference on 614 Platform Technology and Service (PlatCon). doi: 10.1109/platcon.2017.7883724

615 Abbasi A, He M (2019) Convolutional Neural Network with PCA and Batch Normalization for 616 Hyperspectral Image Classification. IGARSS 2019 - 2019 IEEE International Geoscience and 617 Remote Sensing Symposium. doi: 10.1109/igarss.2019.8899329

618 Hayat H, Yazhou Liu, Shah M, Ahmad A (2017) Batch regularization to converge the deep neural 619 network for indoor RGBD scene understanding. 2017 2nd International Conference on Image, 620 Vision and Computing (ICIVC). doi: 10.1109/icivc.2017.7984665

621 Dubey V, Katarya R (2020) An Analysis of Machine Learning Techniques for Flood Mitigation. 622 Advances in Intelligent Systems and Computing 299-307. doi: 10.1007/978-981-15-5148-2_27

623 El-Magd S, Pradhan B, Alamri A (2021) Machine learning algorithm for flash flood prediction 624 mapping in Wadi El-Laqeita and surroundings, Central Eastern Desert, Egypt. Arabian Journal of 625 Geosciences. doi: 10.1007/s12517-021-06466-Z

626 Khan T, Alam M, Shahid Z, Su'Ud M (2020) Investigation of Flash Floods on Early Basis: A

627 Factual Comprehensive Review. IEEE Access 8:19364-19380. doi: 10.1109/access.2020.2967496

628 Yan J, Jin J, Chen F, Yu G, Yin H, Wang W (2017) Urban flash flood forecast using support 629 vector machine and numerical simulation. Journal of Hydroinformatics 20:221-231. doi: $63010.2166 /$ hydro.2017.175

631 Liu J, Xiong J, Cheng W, Li Y, Cao Y, He Y, Duan Y, He W, Yang G (2021) Assessment of 632 Flood Susceptibility Using Support Vector Machine in the Belt and Road Region. doi: 633 10.5194/nhess-2021-80

634 Somvanshi M, Chavan P, Tambade S, Shinde S (2016) A review of machine learning techniques 635 using decision tree and support vector machine. 2016 International Conference on Computing 636 Communication Control and automation (ICCUBEA). doi: 10.1109/iccubea.2016.7860040

637 Charbuty B, Abdulazeez A (2021) Classification Based on Decision Tree Algorithm for 638 Machine Learning. Journal of Applied Science and Technology Trends 2:20-28. doi: $63910.38094 /$ jastt20165

640 Dutt M, Nunavath V, Goodwin M (2018) A Multi-layer Feed Forward Neural Network 641 Approach for Diagnosing Diabetes. 2018 11th International Conference on Developments 642 in eSystems Engineering (DeSE). doi: 10.1109/dese.2018.00060

643 Abiodun O, Kiru M, Jantan A, Omolara A, Dada K, Umar A, Linus O, Arshad H, 644 Kazaure A, Gana U (2019) Comprehensive Review of Artificial Neural Network 645 Applications to Pattern Recognition. IEEE Access 7:158820-158846. doi: 646 10.1109/access.2019.2945545 
647 Subeesh A, Kumar P, Chauhan N (2018) Flood Early Detection System Using Internet of 648 Things and Artificial Neural Networks. International Conference on Innovative 649 Computing and Communications 297-305. doi: 10.1007/978-981-13-2324-9_30

650 Kwasigroch A, Grochowski M, Mikolajczyk M (2019) Deep neural network architecture 651 search using network morphism. 2019 24th International Conference on Methods and 652 Models in Automation and Robotics (MMAR). doi: 10.1109/mmar.2019.8864624

653 Liu W, Wang Z, Liu X, Zeng N, Liu Y, Alsaadi F (2017) A survey of deep neural 654 network architectures and their applications. Neurocomputing 234:11-26. doi: 655 10.1016/j.neucom.2016.12.038 